\title{
Reduced ultraviolet-induced DNA damage and apoptosis in human skin with topical application of a photolyase-containing DNA repair enzyme cream: Clues to skin cancer prevention
}

\author{
ENZO BERARDESCA ${ }^{1}$, MARCO BERTONA ${ }^{2}$, KARMELA ALTABAS $^{3}$, \\ VELIMIR ALTABAS ${ }^{3}$ and ENZO EMANUELE ${ }^{2}$
}

${ }^{1}$ San Gallicano Dermatological Institute, IRCCS, Rome; ${ }^{2}$ Department of Health Sciences, University of Pavia,
Pavia, Italy; ${ }^{3}$ Department of Internal Medicine, Clinical Hospital 'Sestre Milosrdnice', Zagreb, Croatia

Received August 23, 2011; Accepted November 3, 2011

DOI: $10.3892 / \mathrm{mmr} .2011 .673$

\begin{abstract}
The exposure of human skin to ultraviolet radiation (UVR) results in the formation of DNA photolesions that give rise to photoaging, mutations, cell death and the onset of carcinogenic events. Photolyase (EC 4.1.99.3) is a DNA repair enzyme that reverses damage caused by exposure to UVR. We sought to investigate whether addition of photolyase enhances the protection provided by a traditional sunscreen (SS), by reducing the in vivo formation of cyclobutane-type pyrimidine dimers (CPDs) and UVR-induced apoptosis in human skin. Ten volunteers (Fitzpatrick skin type II) were exposed to solar-simulated (ss) UVR at a three times minimal erythema dose for 4 consecutive days. Thirty minutes prior to each exposure, the test materials [vehicle, SS (sun protection factor 50) alone, and SS plus photolyase from Anacystis nidulans] were applied topically to three different sites. One additional site was left untreated and one received ssUVR only. Biopsy specimens were taken $72 \mathrm{~h}$ after the last irradiation. The amount of CPDs and the extent of apoptosis were measured by ELISA. Photolyase plus SS was superior to SS alone in reducing both the formation of CPDs and apoptotic cell death (both $\mathrm{P}<0.001$ ). In conclusion, the addition of photolyase to a traditional SS contributes significantly to the prevention of UVR-induced DNA damage and apoptosis when applied topically to human skin.
\end{abstract}

\section{Introduction}

The exposure of human skin to solar ultraviolet radiation (UVR), in particular ultraviolet-B (UVB; 290-320 nm), results in the formation of DNA photolesions that give rise

Correspondence to: Dr Enzo Emanuele, Department of Health Sciences, University of Pavia, Via Bassi 21, I-27100 Pavia, Italy E-mail: enzo.em@libero.it

Key words: photolyase, ultraviolet radiation, skin, cyclobutane-type pyrimidine dimers, apoptosis, skin cancer to photoaging, mutations, cell death and the onset of carcinogenic events $(1,2)$. Previous studies have shown that the UVB component of solar radiation induces the formation of two major photoproducts: cis-syn cyclobutane pyrimidine dimers (CPDs) and (6-4) pyrimidine-pyrimidone photoproducts $(3,4)$. Among the DNA photoproducts produced upon UVB absorption by DNA, CPDs - formed from the photo [2+2] cycloaddition of the 5,6-double bond of two adjacent pyrimidine nucleotides - are predominant $(5,6)$. CPDs interrupt normal replication and DNA transcription processes and may ultimately predispose to skin cancer (5). Evidence also suggests that severely UVB-damaged cells undergo apoptosis in favor of surrounding normal cells. Accordingly, UVB-mediated apoptosis is currently regarded as a protective mechanism preventing malignant transformation by eliminating cells that carry high loads of UVB-induced DNA damage $(3,7)$.

Photoprotection is the primary preventive and therapeutic strategy against UVR-induced DNA damage and skin cancer $(8,9)$. Apart from traditional behavioral measures, i.e., wearing sun-protective clothes, reducing sun exposure to a minimum and using sunscreen products (8), an innovative approach to the vexing clinical issue of photoprotection is topical application of xenogenic DNA repair enzymes (10). Two different methods have recently been established: the use of T4 endonuclease V and the application of photolyase. The use of T4 endonuclease $\mathrm{V}$ has been shown to be clinically useful in protecting patients with a nucleotide excision repair defect from premalignant and malignant skin lesions (11). On the other hand, application of photolyase, a xenogenic enzyme, which has been found in different organisms, is also capable of removing UVB-induced CPDs from normal human skin cells in vivo and appears to be more effective than $\mathrm{T} 4$ endonuclease $\mathrm{V}$ in damage repair (10).

Photolyase (EC 4.1.99.3) is a monomeric DNA repair flavoenzyme of 50-60 kDa, which reverses damage caused by exposure to UVR $(12,13)$. This enzyme occurs in almost all living organisms exposed to sunlight, the only exception being placental mammals, such as humans (14). Previous in vitro studies have shown that CPDs are effectively repaired by photolyase by means of a catalytic photocycle, termed photoreactivation, that uses blue light energy (15). Photoreactivation can be thus defined as the reversal of the harmful effects of 
UVR by concomitant or subsequent exposure of the organism to near-UV/blue light (300-500 nm; maximal action spectrum: 430-460 nm) (12,16). Photolyase from the cyanobacterium Anacystis nidulans contains a light-harvesting chromophore, 8-hydroxy-5-deazaflavin (8-HDF), and flavin adenine dinucleotide (FAD), which is crucial for catalytic activity (17). In a previous clinical study, Stege et al (18) showed that the topical application of liposome formulations containing Anacystis nidulans-derived photolyase onto human skin provide protection against UVB-induced damage, such as erythema and immunogenic hypersensitivity reactions. Based on this premise, we hypothesized that the addition of photolyase may improve the protection provided by a traditional sunscreen (SS) by reducing the formation of CPDs at the DNA level and preventing UVR-induced apoptosis. To test this possibility, we compared the molecular effects of topical preparations containing a broad-spectrum SS alone (sun protection factor 50) and a combination of both SS and photolyase in human skin in vivo.

\section{Materials and methods}

Subjects. Ten healthy Caucasian volunteers (5 males and 5 females, age range 25-36 years) with Fitzpatrick skin type II were included in this study. Subjects with a history of photodermatosis and skin cancer were excluded. No subject was taking any photosensitizing or anti-inflammatory medications. The study was conducted in winter to minimize the effect of ambient sun exposure. Institutional Review Board approval and informed consent were obtained from all participants.

Test materials. Test materials were supplied by Biodue S.p.A. (Tavarnelle Val Di Pesa, Italy). The SS (sun protection factor 50) contained Tinosorb M, 50\% solution (4\%), Parsol MCX (8\%), Tinosorb S (5\%), Eusolex 9020 (2\%) and Eusolex OCR (1\%). The photolyase preparation contained the same filters plus photolyase derived from the cyanobacterium Anacystis nidulans in a liposomal preparation (1\%). The vehicle was a commercially available moisturizer base.

Solar simulator. Solar-simulated radiation was produced by an Oriel solar simulator (Model 81292; L.O.T. Oriel, Leatherhead, UK) containing a $1 \mathrm{~kW}$ xenon arc lamp with two dichroic mirrors, a collimator and a 1-mm WG320 filter. The optical design of this particular solar simulator gives a field of even irradiance $(290-400 \mathrm{~nm})$ to the skin surface when positioned $11 \mathrm{~cm}$ from the source, of which $\sim 10 \%$ is UVB $(280-320 \mathrm{~nm})$ and the remainder UVA. The spectral irradiance was measured with an OL754 spectroradiometer (Optronics, Orlando, FL, USA), calibrated for wavelength and intensity against standard lamps. The spectroradiometer was used to calibrate a handheld IL700 radiometer (International Light, Newburyport, MA, USA), which was then used to rapidly monitor lamp output on a daily basis.

Irradiation and treatment protocol. Two weeks prior to the test irradiations, the minimal erythema dose (MED) was determined for each individual for solar-simulated UVR (290-400 nm) and expressed in $\mathrm{mJ} / \mathrm{cm}^{2}$ using a light-proof adhesive-backed foil template that was sequentially uncovered
Table I. Treatment description of the five experimental sites

\begin{tabular}{|c|c|c|c|}
\hline Site & Condition & $\begin{array}{c}\text { Solar-simulated } \\
\text { UVR }\end{array}$ & Category \\
\hline 1 & Baseline & - & Reference \\
\hline 2 & UVR only & + & $\begin{array}{l}\text { UVR only } \\
\text { positive control }\end{array}$ \\
\hline 3 & Vehicle & + & Vehicle + UVR \\
\hline 4 & Sunscreen alone & + & Sunscreen + UVR \\
\hline 5 & $\begin{array}{l}\text { Sunscreen plus } \\
\text { photolyase }\end{array}$ & + & $\begin{array}{l}\text { Sunscreen + } \\
\text { photolyase + UVR }\end{array}$ \\
\hline
\end{tabular}

to deliver quantities of UV above and below the expected MED of skin phototype II individuals for solar-simulating UVR. The sites were examined $24 \mathrm{~h}$ after irradiation and the MED was determined as the site that showed minimal, uniform perceptible erythema. Before irradiation, five circular areas (10 $\mathrm{mm}$ diameter) were marked out on the non-exposed lower back of each participant (Table I). On four consecutive days, four sites (designated sites 2-5) were exposed to solarsimulated (ss) UVR at three times MED. Site 1 received no ssUVR (reference). Thirty minutes prior to each irradiation, the following products were applied to sites 3-5, respectively: vehicle (moisturizer base cream), SS alone and SS plus photolyase. No product was applied to site 2 (UVR only). Subjects reported to the study center for all irradiations, and all test product applications were performed by the investigators. Seventy-two hours following the last exposure to ssUVR, skin specimens were obtained through a $4-\mathrm{mm}$ punch biopsy from all sites for molecular analyses.

DNA extraction. The skin biopsy specimens were cleaved in half, and one piece was thawed at room temperature, minced and lysed by 3 cycles of freezing (in an ethanol-dry-ice bath) and thawing (at $95^{\circ} \mathrm{C}$ ). Samples were digested for $12 \mathrm{~h}$ at $60^{\circ} \mathrm{C}$ with proteinase $\mathrm{K}$ in $100 \mathrm{mmol} / 1$ Tris- $\mathrm{HCl}(\mathrm{pH} \mathrm{7.4)}$, $150 \mathrm{mmol} / \mathrm{l} \mathrm{NaCl}$ and $10 \mathrm{mmol} / \mathrm{l} \mathrm{EDTA}$ (pH 8.0). Proteinase K was heat inactivated at $95^{\circ} \mathrm{C}$ for $10 \mathrm{~min}$, and homogenates were extracted using the Puregene DNA Isolation kit (Gentra Systems, Minneapolis, MN, USA). The kit contains two main reagents: cell lysis and protein precipitation solutions. In brief, DNA was extracted from homogenates using a lysis buffer solution and then treated with RNase A. The kit removes proteins using a precipitation solution, followed by 2-propanol to pellet the DNA.

ELISA for CPDs. Determination of CPDs in DNA extracted from homogenates was performed by ELISA, as previously described (13). Briefly, purified DNA was diluted to $0.6 \mu \mathrm{g} / \mathrm{ml}$ in $2 \mathrm{X}$ saline-sodium citrate (SSC) buffer, and then denatured by boiling for $5 \mathrm{~min}$. Samples were placed on ice, and $100 \mu \mathrm{l}$ of each was added to $1 \%$ protamine sulfate-treated wells in 96-well ELISA plates. The primary antibody (catalog no. MC-062; Kamiya Biomedical Company, Seattle, WA, USA) was diluted $1: 2,000$ to $0.25 \mu \mathrm{g} / \mathrm{ml}$ in $0.05 \%$ Tween-20/0.25\% bovine serum albumin (BSA)/1X phosphate-buffered saline (PBS) blocking solution. An alkaline phosphatase conjugate 


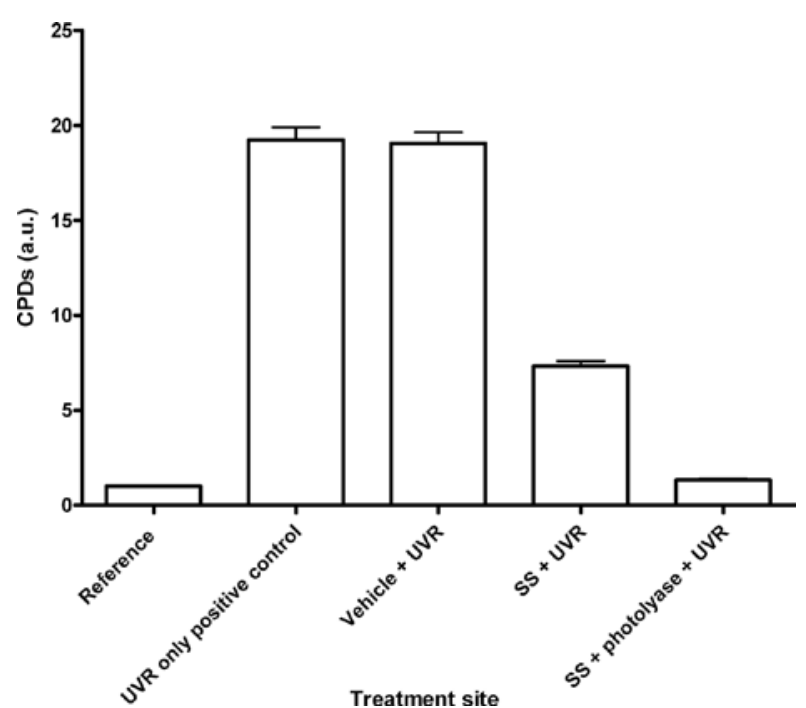

Figure 1. Effect of a sunscreen (SS) with or without photolyase on CPD formation after repetitive ultraviolet radiation (UVR) exposure. ANOVA followed by Newman-Keuls tests was used to analyze CPDs. Repetitive irradiation significantly increased the formation of CPDs in both UVR only positive control and vehicle + UVR sites $(\mathrm{P}<0.001$ vs. baseline). SS alone significantly, but not completely, prevented CPD formation $(\mathrm{P}<0.001$ vs UVR only positive control and vehicle + UVR sites). However, topical SS + photolyase was significantly better than SS alone $(\mathrm{P}<0.001)$.

(catalog no. AP130A; Chemicon, Temecula, CA, USA) diluted $1: 10,000$ in blocking solution was used as secondary antibody. Following incubation with the secondary antibody, wells were washed with $1 \mathrm{X}$ PBS, and CPDs were quantified with a nitrophenyl phosphate disodium-substrate assay. Absorbance was read at $405 \mathrm{~nm}$. Each experiment was performed in duplicate, and the results were averaged. Higher absorbance is associated with higher CPDs. The results were plotted in arbitrary units relative to the values of the baseline control site.

Assessment of apoptosis. Apoptosis in skin homogenates was measured using a colorimetric Cell Death Detection ELISAPlus assay, according to the manufacturer's instructions (Roche Diagnostics, Basel, Switzerland). The principle of this test was based on the detection of mono- and oligonucleosomes in the cytoplasmic fractions of cell lysates using biotinylated antihistone- and peroxidase-coupled anti-DNA antibodies. Absorbance was read at $405 \mathrm{~nm}$. Higher absorbance is associated with increased apoptosis. The enrichment factor was used as a parameter of apoptosis and is shown on the y-axis as the means \pm standard deviation from experiments performed in duplicate (19). An enrichment factor of 1 was deemed to represent background or spontaneous apoptosis in the baseline control site.

Statistical analysis. All calculations were performed in GraphPad Prism 4.0 (GraphPad Inc., San Diego, CA, USA) and SPSS 14.0 (SPSS Inc., Chicago, IL, USA). Data were checked for normal distribution using the Shapiro-Wilk test. All variables had a Gaussian distribution, and parametric analyses were thus exploited. Variables were expressed as the means and standard deviations or counts, as appropriate. One-way ANOVA followed by Newman-Keuls multiple-comparison post hoc test was used to analyze intergroup differences. Given

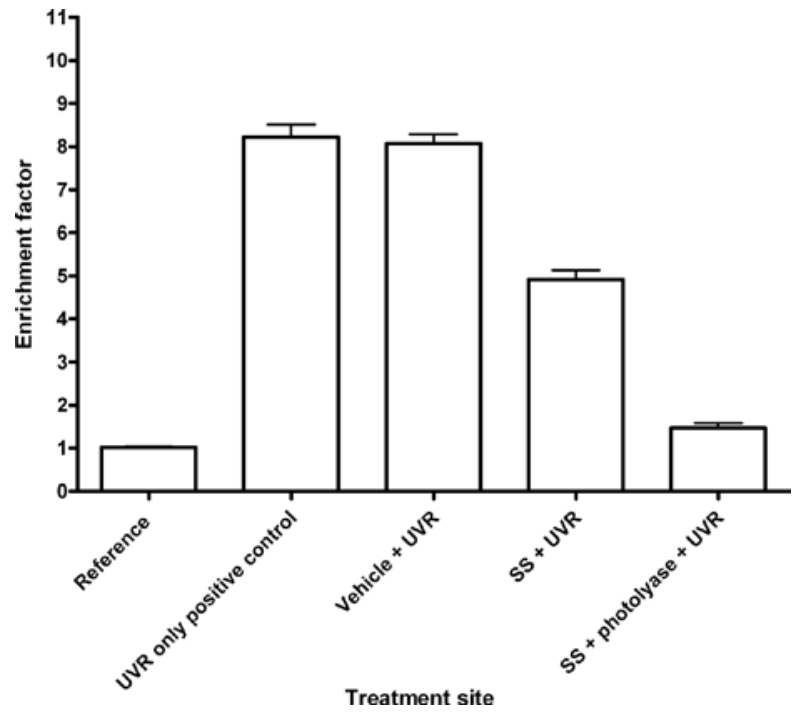

Figure 2. Effect of a sunscreen (SS) with or without photolyase on apoptosis in skin biopsies after repetitive ultraviolet radiation (UVR) exposure. ANOVA followed by Newman-Keuls tests was used to analyze apoptosis. Repetitive irradiation significantly increased apoptosis in both the UVR only positive control and vehicle + UVR sites $(\mathrm{P}<0.001$ vs. baseline). SS alone significantly, but not completely, prevented apoptosis $(\mathrm{P}<0.001 \mathrm{vs}$. UVR only positive control and vehicle + UVR sites). However, topical SS + photolyase was significantly better than $\mathrm{SS}$ alone $(\mathrm{P}<0.001)$.

the exploratory nature of the study, no Bonferroni correction was used. Statistical significance was defined at $\mathrm{P}<0.05$ using two-sided tests.

\section{Results}

The mean MED for solar-simulated UVR was $59 \pm 10 \mathrm{~mJ} / \mathrm{cm}^{2}$. The MEDs were recorded for each individual and the experimental schedule was initiated using the solar simulator based on the initial MED for each individual.

$C P D s$. The effect of a sunscreen with or without photolyase on CPD formation after repetitive UVR exposure on human skin in vivo is depicted in Fig. 1. Repetitive irradiation significantly increased the formation of CPDs in both the UVR only positive control and vehicle + UVR sites (19-fold higher at both sites compared to baseline, $\mathrm{P}<0.001)$. SS alone significantly, but not completely, prevented CPD formation, reducing it by $62 \%$ $(\mathrm{P}<0.001$ vs. UVR only positive control and vehicle + UVR sites). However, topical SS + photolyase prevented CPD formation by $\sim 93 \%$, and was thus better than SS alone $(\mathrm{P}<0.001)$.

Apoptosis. Cell Death Detection ELISAPlus was used to quantify DNA fragmentation in apoptotic cells based on the detection of mono- and oligonucleosomes in the cytoplasmic fractions of cell lysates obtained from skin biopsies under different experimental conditions. As shown in Fig. 2, repetitive irradiation significantly promoted apoptosis in both UVR only positive control and vehicle + UVR sites (8.1-fold higher at both sites compared to baseline, $\mathrm{P}<0.001)$. SS alone significantly, but not completely, prevented CPD formation, reducing it by $40 \%$ $(\mathrm{P}<0.001$ vs. UVR only positive control and vehicle + UVR 
sites). However, topical SS + photolyase prevented apoptosis by $\sim 82 \%$, and, hence, was better than SS alone $(\mathrm{P}<0.001)$.

\section{Discussion}

Photoreactivation, in which visible light is used to counteract the deleterious effects of DNA damage produced by UVR, is found in plants and several photosynthetic microorganisms, but not in human beings (20). In the South American opossum (Monodelphis domestica), which possess an endogenous CPD photolyase, photoreactivation has been shown to clearly protect the skin and eye from several immunological and cytotoxic effects of UVR (21). More recently, the topical application of photolyase-containing liposomes has been shown to provide a higher level of protection to the UVR-exposed human skin (18).

The data from this study suggest that the addition of photolyase to a traditional SS significantly improves the protection offered by topical creams against both ssUVR-induced DNA damage and apoptotic cell death in the skin. Notably, the in vivo model used in this study consisted of repeated ssUVR exposure. As the accumulation of residual DNA damage via repeated ssUVR exposure is deemed to play a key role in the development of skin cancer (22), our results clearly indicate that photolyase-containing topical preparations are superior to traditional sunscreens in reducing cancerous and precancerous skin lesions as well as photoaging.

Our results not only confirm, but also substantially expand previous findings on the potential usefulness of photolyase for human photoprotection. Decome et al (15) previously showed that UVA-photoactivated photolyases included in liposomes efficiently repaired CPDs and decreased single-strand break levels by 2.6 to 3.3 -fold following a single dose of UVB in human keratinocytes. In humans, topical photolyasecontaining preparations has, to date, only been tested in a limited number of studies. Stege et al have demonstrated that topical application of photolyase containing liposomes in vivo to UVB-irradiated skin and subsequent exposure to photoreactivating light decreased the number of UVB radiation-induced dimers by $45-50 \%$ (18). Of note, the protocol used by Stege et al was significantly different from the current one. First, in their experiments Stege et al used photolyasecontaining liposomes without the addition of a SS. Second, in the present study we employed a multiple irradiation protocol, which more strictly mimics long-term sunlight exposure (23). These differences in experimental design and in the preparations used may explain the higher protective effect of photolyase-containing creams against the formation of CPDs in vivo. Indeed, the combination of $\mathrm{SS}$ and photolyase almost completely abrogated ssUVR-induced CPDs. Furthermore, removal of CPDs from ssUVR-irradiated human skin through the application of SS plus photolyase resulted in a relatively high level of protection against ssUVR-induced apoptotic cell death in human skin. In general, UVR not only induces DNA damage in epidermal cells, but it also interferes with skin homeostasis, which is maintained by a unique distribution pattern of apoptosis-inducing and -preventing molecules (24). If the DNA damage is not repaired or the damaged cells are not eliminated by apoptosis, this can lead to cell transformation, uncontrolled proliferation and eventually skin tumor formation (24). In this context, apoptosis provides an efficient safeguard mechanism against cancer $(25,26)$. As expected, we found that apoptosis increased markedly after repeated ssUVR irradiation. However, when the skin was pre-treated with SS plus photolyase, the apoptotic process, which is the result of direct DNA photodamage, was reduced by $82 \%$. The present study therefore suggests that the addition of photolyase to a SS protects against ssUVR-induced phototoxicity by inhibiting DNA damage and the associated onset of apoptotic cell death.

Several caveats of this study merit comment. The sample size was relatively small, and replication in a larger population is necessary. Second, the study group was ethnically homogenous, therefore the results may not apply to other populations. Third, we cannot rule out the possibility that a higher amount of sunscreen alone applied to the skin could further reduce the formation of CPDs in vivo. However, when the study was designed, we decided to apply a rigorous protocol to allow comparability with previous studies. The application density of the sunscreen formulation used in this study is that used for routine SPF assessment $\left(2 \mathrm{mg} / \mathrm{cm}^{2}\right)$ and is in line with previous studies in the field of sunscreen photoprotection $(27,28)$. Whether a higher application density of the SS alone could further reduce CPD formation and skin apoptosis in vivo is outside the scope of our report, but deserves further scrutiny. Fourth, this study focused solely on the molecular effects of photolyase. As a consequence, we do not have direct comparative data between photolyase and other enzymatic DNA repair systems like T4 endonuclease V. The DNA-repairing mechanism of action of $\mathrm{T} 4$ endonuclease $\mathrm{V}$ differs significantly from that of photolyase (19). T4 endonuclease $\mathrm{V}$ generates a single-stranded incision in DNA at the site of a CPD in a lightindependent fashion. The mechanism of incision involves the sequential action of two independent activities of endonuclease V: a DNA glycosylase that cleaves the glycosylic bond of the 5'-pyrimidine of a dimer and an apyrimidinic endonuclease that cleaves the phosphodiester bond between the two pyrimidines (11).

These limitations notwithstanding, this study demonstrates that topical photolyase added to a SS reduces ssUVR-mediated DNA damage and apoptosis even at low, cosmetically usable, concentrations, suggesting that this strategy may be applied for photochemoprevention. Hence, the present findings provide considerable insight into the prevention of human skin cancers.

\section{References}

1. Marrot L and Meunier JR: Skin DNA photodamage and its biological consequences. J Am Acad Dermatol 58 (Suppl 2): 139-148, 2008.

2. Ichihashi M, Ueda M, Budiyanto A, Bito T, Oka M, Fukunaga M, Tsuru K and Horikawa T: UV-induced skin damage. Toxicology 189: 21-39, 2003.

3. Batista LF, Kaina B, Meneghini R and Menck CF: How DNA lesions are turned into powerful killing structures: insights from UV-induced apoptosis. Mutat Res 681: 197-208, 2009.

4. Rastogi RP, Richa, Kumar A, Tyagi MB and Sinha RP: Molecular mechanisms of ultraviolet radiation-induced DNA damage and repair. J Nucleic Acids 2010: 592980, 2010.

5. Vink AA and Roza L: Biological consequences of cyclobutane pyrimidine dimers. J Photochem Photobiol B 65: 101-104, 2001.

6. Durbeej B and Eriksson LA: On the formation of cyclobutane pyrimidine dimers in UV-irradiated DNA: Why are thymines more reactive? Photochem Photobiol 78: 159-167, 2003. 
7. Assefa Z, van Laethem A, Garmyn $M$ and Agostinis $P$ Ultraviolet radiation-induced apoptosis in keratinocytes: on the role of cytosolic factors. Biochim Biophys Acta 1755: 90-106, 2005.

8. Lautenschlager S, Wulf HC and Pittelkow MR: Photoprotection. Lancet 370: 528-537, 2007.

9. Wang SQ, Balagula Y and Osterwalder U: Photoprotection: a review of the current and future technologies. Dermatol Ther 23: 31-47, 2010.

10. Stege H: Effect of xenogenic repair enzymes on photoimmunology and photocarcinogenesis. J Photochem Photobiol B 65 105-108, 2001.

11. Zahid S and Brownell I: Repairing DNA damage in xeroderma pigmentosum: T4N5 lotion and gene therapy. J Drugs Dermatol 7 : 405-408, 2008.

12. Thoma F: Light and dark in chromatin repair: repair of UV-induced DNA lesions by photolyase and nucleotide excision repair. EMBO J 18: 6585-6598, 1999.

13. Sancar A: Structure and function of photolyase and in vivo enzymology: 50th anniversary. J Biol Chem 283: 32153-32157, 2008.

14. Garinis GA, Jans J and van der Horst GT: Photolyases: capturing the light to battle skin cancer. Future Oncol 2: 191-199, 2006.

15. Decome L, De Méo M, Geffard A, Doucet O, Duménil G and Botta A: Evaluation of photolyase (Photosome) repair activity in human keratinocytes after a single dose of ultraviolet B irradiation using the comet assay. J Photochem Photobiol B 79: 101-108, 2005.

16. Weber S: Light-driven enzymatic catalysis of DNA repair: a review of recent biophysical studies on photolyase. Biochim Biophys Acta 1707: 1-23, 2005.

17. Kort R, Komori H, Adachi S, Miki K and Eker A: DNA apophotolyase from Anacystis nidulans: $1.8 \AA$ structure, 8 -HDF reconstitution and X-ray-induced FAD reduction. Acta Crystallogr D Biol Crystallogr 60: 1205-1213, 2004.

18. Stege H, Roza L, Vink AA, Grewe M, Ruzicka T, Grether-Beck S and Krutmann J: Enzyme plus light therapy to repair DNA damage in ultraviolet-B-irradiated human skin. Proc Natl Acad Sci USA 97: 1790-1795, 2000
19. Yarosh DB, Boumakis S, Brown AB, Canning MT, Galvin JW, Both DM, Kraus E, O'Connor A and Brown DA: Measurement of UVB-induced DNA damage and its consequences in models of immunosuppression. Methods 28: 55-62, 2002.

20. Sancar GB: Enzymatic photoreactivation: 50 years and counting. Mutat Res 451: 25-37, 2000

21. Ley RD, Applegate LA, Fry RJ and Sanchez AB: Photoreactivation of ultraviolet radiation-induced skin and eye tumors of Monodelphis domestica. Cancer Res 51: 6539-6542, 1991.

22. Aragane Y, Kulms D, Metze D, Wilkes G, Pöppelmann B, Luger TA and Schwarz T: Ultraviolet light induces apoptosis via direct activation of CD95 (Fas/APO-1) independently of its ligand CD95L. J Cell Biol 140: 171-182, 1998.

23. Wu Y, Matsui MS, Chen JZ, Jin X, Shu CM, Jin GY, Dong GH, Wang YK, Gao XH, Chen HD and Li YH: Antioxidants add protection to a broad-spectrum sunscreen. Clin Exp Dermatol 36: 178-187, 2011.

24. Erb P, Ji J, Wernli M, Kump E, Glaser A and Büchner SA: Role of apoptosis in basal cell and squamous cell carcinoma formation. Immunol Lett 100: 68-72, 2005.

25. Erb P, Ji J, Kump E, Mielgo A and Wernli M: Apoptosis and pathogenesis of melanoma and nonmelanoma skin cancer. Adv Exp Med Biol 624: 283-295, 2008.

26. Massari LP, Kastelan M and Gruber F: Epidermal malignant tumors: pathogenesis, influence of UV light and apoptosis. Coll Antropol 31 (Suppl 1): 83-85, 2007.

27. De Fine Olivarius F, Wulf HC, Crosby J and Norval M: Sunscreen protection against cis-urocanic acid production in human skin. Acta Derm Venereol 79: 426-430, 1999.

28. Bodekaer M, Faurschou A, Philipsen PA and Wulf HC: Sun protection factor persistence during a day with physical activity and bathing. Photodermatol Photoimmunol Photomed 24: 296-300, 2008 